\section{The impact of the National HIV Health Care Worker Hotline}

To the Editor: The National HIV Health Care Worker (HCW) Hotline was established in 2008, in collaboration with the Foundation for Professional Development (FPD) and PEPFAR/USAID, to support the safe and effective roll-out of antiretroviral treatment in South Africa. It is based in the Medicines Information Centre, Division of Clinical Pharmacology, UCT, and has access to the latest information and numerous clinical experts.

The toll-free hotline operates weekdays, $8 \mathrm{~h} 30$ - 16h30, providing up-to-date information to all health care workers on aspects concerning the treatment of HIV infection and related diseases. Use of the service has consistently increased to over 300 calls a month.

HCWs who called the hotline in August and September 2009 with patient-specific queries were asked to complete a standardised questionnaire which evaluated whether the information provided was used and how this affected patient care. The most frequent users of the hotline were doctors (69\%), pharmacists (14\%) and nurses (11\%), which may reflect the fact that doctors remain predominant in decisions around HIV care.

Of those who called the hotline with a clinical query, $96 \%$ reported that they changed their patient management as a result of the information provided. Most actions that were a consequence of the advice given concerned direct treatment-related decisions; these included treatment initiation (34\%), dose adjustment (19\%), discontinuation (44\%) and change of ART. A substantial proportion of actions concerned the overall management of patients, such as the initiation of further diagnostic procedures, referrals to specialist services and hospital admissions. These figures demonstrate that the hotline is used for medicine-specific questions and the overall management of patients. Almost all callers interviewed confirmed that the information provided was useful, timely and of benefit to the patient.

The public health sector aims to substantially increase the number of HIV-infected individuals receiving ART. Nurse-initiated management of ART (NIMART) is a goal of the Department of Health, ${ }^{1}$ and nurses will increasingly initiate and manage patients.

We conclude that a telephonic helpline, such as the HIV HCW Hotline, providing clinical advice and support, is a valuable resource for supporting all health care workers in South Africa.

\section{Annoesjka Swart \\ Briony Chisholm \\ Medicines Information Centre \\ University of Cape Town \\ briony.chisholm@uct.ac.za}

\section{Karen Cohen}

Marc Blockman

Division of Clinical Pharmacology

University of Cape Town

\section{Hans-Friedemann Kinkel}

HIV \& TB Medicine Unit

Foundation for Professional Development

Pretoria

\section{Tamara Kredo}

South African Cochrane Centre

$M R C$

Cape Town

1. South African National Department of Health. Clinical Guidelines for the Management of HIV and AIDS in Adults and Adolescents, 2010. http://www.doh.gov.za/docs/facts-f.html (accessed 1 Novembe 2010).

\section{A South African doctors' team in the World Cup?}

To the Editor: I wish to bring to the attention of readers an opportunity for them to play international football in the next medical world cup! How would you like to play against Brazil, Germany or Great Britain?

For many, football is a passion that begins shortly after taking their first steps. Medicine is another passion, finding expression later in life. These two drives often compete, with usually one prevailing. It is with great pleasure that The World Medical Football Federation seeks to carry on the tradition of uniting medicine and football. The Federation has been growing year after year, and is a truly international social, professional and sporting amalgamation.

I was involved in putting together a group of 20 British doctors in 2006, and we entered the competition in Germany. Since then, we have played annually in Australia, Lithuania, South Korea and Austria. The XVII Global Congress on Medicine and Health in Sport, and World Football Championship of Medical Teams, takes place on 23 - 30 July 2011 in Manchester, UK.

The week involves a medical conference with speakers from each country on some mornings, and the teams taking to the field in the afternoons, playing six 11-a-side matches in 7 days. The football is competitive but, with rules such as 'There must be at least 3 outfield players over the age of 40', also aims to be enjoyable. Many delegates bring their families, and there is a great atmosphere in the hotel and at the daily social activities.

If there are any interested doctors in South Africa who were inspired by the South African World Cup, please contact me for more details about joining us next year in England. We have most of the continents represented, with the Americas, Europe, Asia, and Australasia, but we are missing Africa! Please have a look at our website (www.britishmedicalfootballteam.co.uk) to see what's planned, and consider being part of this fantastic event. I look forward to answering any further questions.

\section{Paul Fourie}

Blackburn, UK

(formerly Springs, RSA)

paul.fourie@nhs.net>

\section{HIV diagnosis in a mobile unit}

To the Editor: I read the article on HIV diagnosis in mobile unit with great interest. ${ }^{1}$ Van Schaik et al. concluded that 'Mobile services are accessed by a different population compared with facility-based services. ${ }^{1}$ I agree that the HIV diagnosis via a mobile unit can be a useful approach for early diagnosis. However, there are some problems to be kept in mind. Quality control (QC) of the HIV test should be carefully considered. There can be problems in this regard in good hospital laboratories, and there is no doubt that QC could potentially be more difficult in a mobile unit. ${ }^{2}$ To optimise the usefulness of the mobile HIV screening programme, standardisation and quality management are very important.

\section{Viroj Wiwanitkit \\ Wiwanitkit House \\ Bangkhae \\ Bangkok \\ Thailand \\ wviroj@yahoo.com}

\footnotetext{
van Schaik N, Kranzer K, Wood R, Bekker LG. Earlier HIV diagnosis - are mobile services the answer? S Afr Med J 2010;100:671-674

Proffitt MR Yen-Lieberman B. Laboratory diagnosis of human immunodeficiency virus infection. Infect Dis Clin North Am 1993:7(2):203-219.
} 
Van Schaik et al. reply: We agree that adequate and regular quality control of HIV test kits as well as adequate training in performing HIV tests are extremely important.

HIV tests performed at the mobile clinic, community clinic and hospital are all rapid HIV tests, performed outside a laboratory by nursing staff, and are subject to the same quality control issues.

Staff working on the mobile unit are all trained in the use of the rapid tests and our standard operating procedures. We have our own quality control programme and are part of an external quality assurance programme run by the National Health Laboratory Service (NHLS), where to date we have scored $100 \%$. In addition we have an informal quality control method in that we see many known HIVpositive individuals who request re-testing. Any discrepant results are confirmed with laboratory HIV enzyme-linked immunosorbent assay (ELISA)/polymerase chain reaction (PCR).

$\mathrm{HIV}$ test kits are stored at temperatures of less than $30^{\circ} \mathrm{C}$ in our offices and only enough for that day's testing are taken out on the mobile unit.

For our own quality control programme, samples from known positive and known negative individuals (confirmed by laboratory HIV ELISA testing) are run with each new batch and monthly thereafter. The control samples must give the intended response (i.e. positive or negative). The staff member who performed the quality control procedure records the results on the rapid HIV testing quality control log sheet, which then gets filed. Any control problems are immediately reported to the project manager and acted on.

\section{Disaster preparedness - looking forward}

To the Editor: I refer to the article 'Haiti: The South African perspective'1 in the SAMJ of August 2010.

It is timeous that the authors have opened the debate on the South African medical rescue response to disasters, particularly at home and on our continent. The problems are succintly set out, real and need to be addressed meaningfully and rapidly. While the challenges are clear, the way forward as suggested in the article is less so. Given the less than organised, if praiseworthy, efforts of the two organisations mentioned in the article, it seems to be compounding the issue by suggesting that the future disaster response be placed under the auspices of the Emergency Medicine Society of South Africa (EMSSA) - another non-statutory body.

South Africa, as one of the most developed countries on the continent, will be expected internationally to respond meaningfully to natural and other disasters in Africa. To be able to do this will at least require government approval and resource support if there is to be a rapid and effective deployment of medical rescue assistance.

At the end of the day, most of the material and human resources required either belong to or work for one or other government department, provincial and national, including a large number of volunteers. In this particular context, I believe the National Department of Health in conjunction with those provinces that possess the requisite assets, which will include departments of emergency medicine, should take the lead. Together they successfully mobilised for the 2010 Soccer World Cup - surely an African disaster response is no less deserving of this kind of effort.

\section{A G Mac Mahon}

Cape Town

alanjean@telkomsa.net
1. Van Hoving DJ, Smith WP, Kramer EB, de Vries S, Docrat F, Wallis LA. Haiti: The South African perspective. S Afr Med J 2010;8:513-515.

\section{Schistosomiasis - an endemic but neglected tropical disease in Limpopo}

To the Editor: After malaria, schistosomiasis is the second most prevalent tropical infection, but is first among the neglected tropical diseases (NTDs). ${ }^{1}$ Worldwide, an estimated 750 million people are at risk of schistosomiasis, and 200 million have the disease; $85 \%$ of the latter and all 20 million with severe disease are concentrated in Africa. ${ }^{2,3}$

We conducted a study that describes the pathology of biopsy diagnosed schistosomiasis, silent or symptomatic, in Limpopo Province to call attention on an NTD with potentially severe morbidity and mortality. This is especially important in view of the HIV/AIDS epidemic that poses a higher burden on women than men. Evidence is accumulating that female genital schistosomiasis (FGS) acts as a co-factor in the genesis of cervical pre-invasive and invasive lesions and/or as an entry point for the HI virus.

Over the period 2008-2009, all new cases of biopsy diagnosed schistosomiasis were prospectively recorded. The diagnosis was made in the presence of viable (embryonated) and/or non-viable (calcified) ova; Schistosoma haematobium in the presence of a terminal spike and S. mansoni if the spike was lateral.

The patients' age, gender, geographical origin, anatomical site and type of lesion, if any, were recorded. There were 266 females, and 45 males (F/M ratio 5.9/1). Table I illustrates the relative distribution by gender and affected organs. FGS accounted for 233 (87.6\%) of the female cases; of the 127 cases involving the cervix, 29 (22.8\%) were HIV-seropositive. Only 44 cases were asymptomatic, namely the incidentally found ova in bilateral tubal ligation $(N=40)$ and prolapsed fibromyomas $(N=4)$. In males, the appendix was the most common site -30 (66.6\%). The overall S. haematobium to S. mansoni ratio was 5.1/1. The ratio was $6.1 / 1$ with urogenital pathology, and 3.2/1 with digestive tract pathology. These figures show an overlap of the two subtypes in urogenital and digestive lesions, and an overall predominance of $S$. haematobium. Of the cases of known geographical origin, $91 \%$ were from the northern and north-eastern parts of the province bordering Zimbabwe and Mozambique.

Failure in the supply of safe water sources and sanitation, and failure to control snail intermediate hosts, lead to the continued transmission of the infection. ${ }^{2,4}$ Control interventions require economic progress, political will and stability, and adequate public health structures and programmes. ${ }^{2}$ School-based health programmes

Table I. Relative distribution by affected organ and gender

\begin{tabular}{lcc}
\hline Organ & Females (266) & Males (45) \\
\hline Upper genital organs* & 74 & \\
Lower genital organs ${ }^{\dagger}$ & 127 & \\
Perineum & 32 & 2 \\
Bowel & 16 & 30 \\
Bladder & 15 & 10 \\
Varia & 2 & 3 \\
"Uterine body, adnexae. & & \\
${ }^{\dagger}$ Cervix. & & \\
\hline
\end{tabular}

